\title{
On Simulating Human Reach Motions for Ergonomics Analyses
}

\author{
Don B. Chaffin \\ Industrial and Operations Engineering and Biomedical Engineering, \\ The University of Michigan, Ann Arbor, MI 48109, U.S.A.
}

\begin{abstract}
Many existing job analysis tools for ergonomics have concentrated on the potential adverse effects of force, posture, and repetition, as these appear to be traditionally recognized risk factors in the workplace. Recent investigations have indicated that this pragmatic approach may be overly simplistic, and thus miss prediction of risk factors associated with certain motions.

This article reviews some of the research under way in the University of Michigan's Human Motion Simulation Laboratory to develop a set of human motion prediction models. To produce these models, over 37,000 motions of 100 men and women from 18 to 78 years in age have been measured with a motion capture system. The motions are typical of people reaching and moving light to moderate load objects while either seated or standing. A 17-link kinematics model has been developed to resolve the dynamics of the motions.

Thus far, initial motion algorithms have been developed that capture well over $95 \%$ of the between participant repeatability. Advantages and limitations of the methods and data being used are discussed and illustrated. (C) 2002 Wiley Periodicals, Inc.
\end{abstract}

\section{INTRODUCTION AND BACKGROUND}

It is only natural that the advent and proliferation of digital computer technologies would stimulate the development of graphical representations of people performing various tasks. The entertainment industry has been a major contributor to the technologies needed to render lifelike human images. A display of one contemporary digital human model (DHM) is depicted in Figure 1. But can these images assist us in designing better workplaces and products? That is the overarching question we face today.

Apparently some companies believe the answer is affirmative. Seven case studies reported in Chaffin (2001) describe successful use of various digital human models to analyze and improve different designs. One of the major reasons for their use seems to be a belief by the designers and managers that using human simulation technology within a digital mock-up (DMU) would decrease the design time and enhance the number and quality of design options that could be rapidly evaluated by the design team. This view is consistent with the concept of reducing the total design and engineering costs by using more computer-aided engineering (CAE) and DMU methods to achieve rapid prototype development and testing, as diagrammed in Figure 2.

In some of the reported cases in Chaffin (2001), simulations of a person in the workplace or vehicle provided the only method of verifying that a design concept was acceptable to a prescribed population, since hardware prototypes were not available (e.g., the International Space Laboratory). 


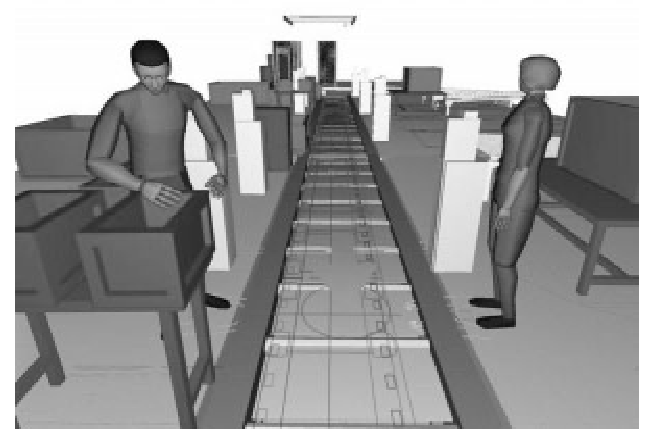

Figure 1 A typical DHM for maximum static reach, fit, and line-of-sight analysis (courtesy of UGS Corporation).

So what types of information about people are the designers interested in having in their DHMs? This question was asked by the SAE G13 Committee of about 250 designers using a 500-question survey form. About 40 of these people responded. These designers showed a strong preference (a score of 4.0 or better on a 5.0 scale) for features such as (a) including different anthropometric data sets and population demographic subgroups; (b) including a variety of clothing, gloves, and helmets; (c) predicting strength and endurance of different populations in a task; (d) simulating realistic motions and postures with minimum task input descriptions, and in both physically constrained and unconstrained conditions; (e) providing hand grip, strength and visual sight lines with and without mirrors and obstructions; (f) providing task timeline analyses; (g) perform-

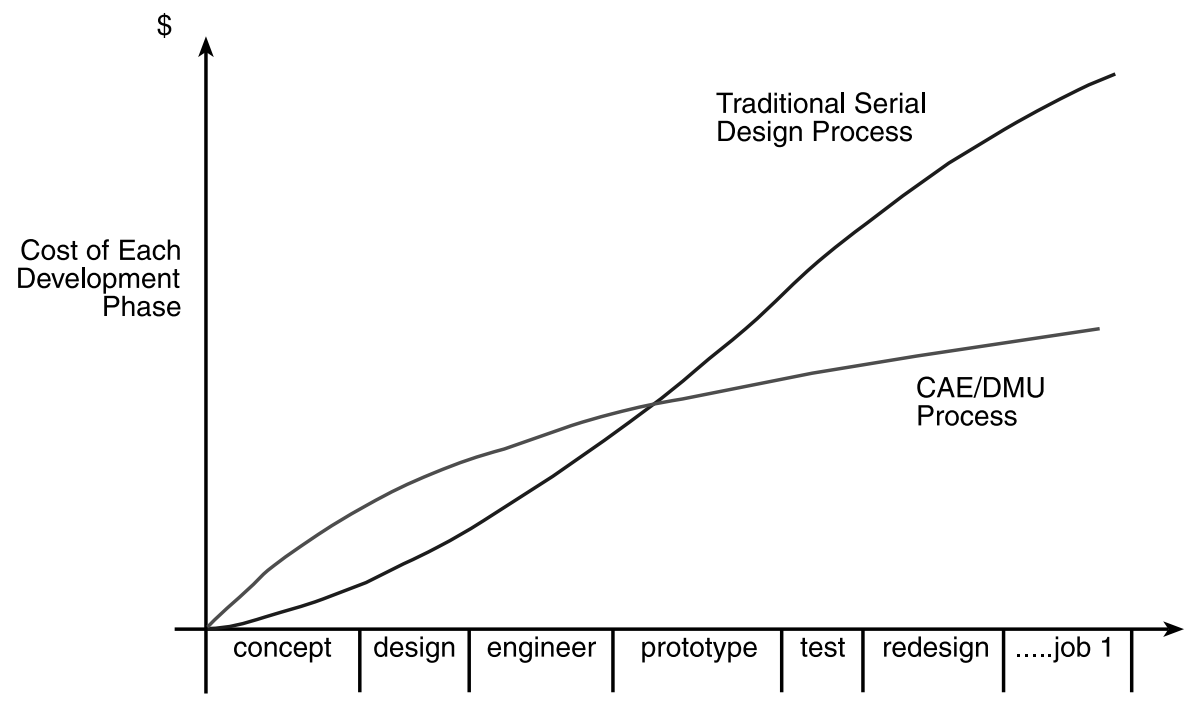

Figure 2 Typical development phases and hypothetical cost profiles believed to exist when using a DMU (with human simulation) early in the design process compared to not using DMUs, which results in increased prototype building and testing costs. 
ing reach and fit analyses for a variety of conditions; and (h) seamlessly accepting I/O commands and data, and/or executing within various computer-aided design (CAD) systems commonly used in rendering and specifying products, tools, and workstations. The results of this survey were compiled by $\mathrm{C}$. Nelson at Boeing Aircraft and are available by contacting the SAE-G13 Committee through www.SAE.org.

What are typical design issues that can be addressed by the existing, commercial, digital human simulation programs? Once again, the seven case studies give us a small glimpse of the answer. It is presented in Table 1 from Chaffin (2001).

This table seems to indicate that the most prevalent use today of digital human modeling is to simulate people of extreme sizes (i.e., to perform three-dimensional anthropometric analyses) for the purpose of providing designs wherein a large variety of people can reach, see, and/or manipulate objects. In a few cases, there existed a need to use a DHM for predicting a population's reach and clearance capability included the mitigating effects of different clothing or personal protective equipment such as heavy gloves or helmets. In some other cases, the issue was one of how much human strength, endurance, or both was required to perform a manual exertion with special concern that the final design comply with federal (NIOSH) or (DOT) policies. In a few cases, the authors believed one of the most important features of a DHM was that the simulations and associated graphics allowed both product and process designers to understand better the potential problems a particular population subgroup could have when operating or servicing a proposed design. This last point was emphasized by some of the authors in that they provided estimates showing that the use of a DHM saved many months and thousands of dollars in design and prototype testing, compared to their traditional methods.

These positive outcomes are encouraging, but the case authors in Chaffin (2001) also indicated the following limitations in the technology (Table 2).

The major limitation appears to be due to the lack of data sharing capabilities, thus requiring a designer to spend a great deal of extra time rendering a workspace with enough detail to perform an analysis. One trend to alleviate this problem seems to be to insert the DHM into a commonly used commercial CAD program. An example of this approach is described by Feyen, Liu, Chaffin, and Jimmerson (2000), wherein the University of Michigan's 3D Static Strength Program was placed in the popular AutoCAD software running in Windows 95. Hopefully, with newer data translators for graphical data, this problem of incompatible data will be alleviated soon.

Another problem revealed in the case studies however, is that the designers were not capable of predicting how a person of certain characteristics should be positioned in the virtual workplace, especially if dynamic motions are of concern. It is this last limitation that has stimulated the development of the Human Motion Simulation Laboratory at the University of Michigan, and the work now to be described.

\section{OBJECTIVES}

What follows is a review of the following issues related to human motion simulation:

1. What are typical human motion modeling methods?

2. What is the intent and scope of the Michigan HUMOSIM Laboratory?

3. What are some common insights gained from the initial motion studies?

4. What research questions are now being pursued? 
TABLE 1. Summary of the Design Issues Successfully Addressed Through the Use of a DHM (from Chaffin, 2000)

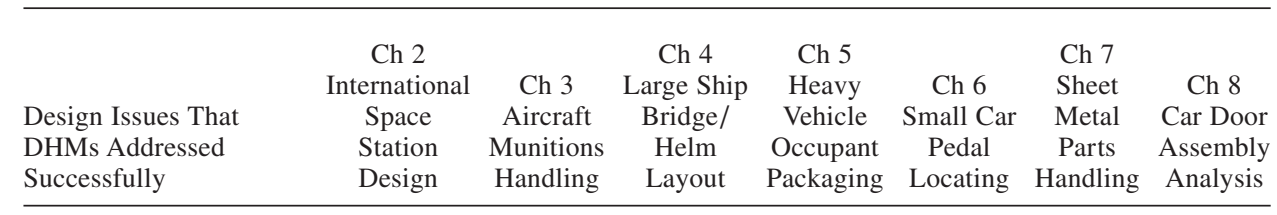

1. Visualize extreme populations (small $\mathrm{F}$ or large $\mathrm{M})$ for reach/visualization

2. Hand clearance for maintenance/ operation

3. Grab handle/step locations

4. Extreme environment (rain/snow)

5. $2+$ person coordination communication for large mock-up analysis

6. Seated vs. standing operation

7. Seated pedal locationsleg postures

8. Population strengthcompare policies of NIOSH, DOT compliance policies

9. Visualizecommunicate ergonomic issues

10. More creative designs'What Ifs'

11. Combined product and manufacturing concurrent engineering

\section{SOME METHODS FOR MODELING HUMAN MOTIONS}

A variety of motion modeling methods have been employed over the years to predict how people reach and move objects. It is beyond the scope of this article to review these methods, but the reader is referred to several excellent reference sources by Allard, Cappozzo, Lundberg, and Vaughan (1998); Nigg, MacIntosh, and Mester (2000); and Zatsiorsky (1998). In essence, these methods fall into the following groups: 
TABLE 2. Summary of Major Limitations in DHM Discussed in Each Case (from Chaffin, 2001)

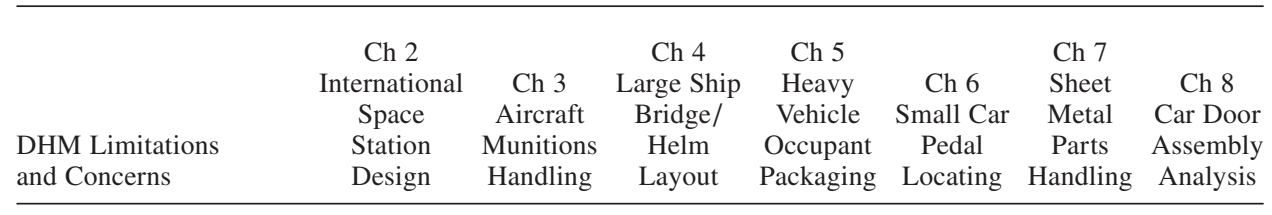

1. Creating 3-D DHM

within a CAD

environment

2. Modeling exertions in extreme postures

3. Deriving postures or motions for dynamic analyses from motion capture files

4. Providing controlforces needed to perform dynamic analyses

5. Estimating postural comfort-for long periods

6. Selecting "critical" tasks

7. Having large-screen 3-D projections for group discussionsdesign reviews

1. Adaptive feed-forward models wherein task-specific motion memory patterns are used to guide the general motion, and then with practice, feedback is used to update the patterns to develop a skilled motion.

2. Control models wherein both postures of some segments and motions of other segments are changed to minimize the deviation from a desired path of motion by changing the gain in select feedback loops.

3. Neural network models in which output data (describing the angles of joints or segment trajectories) are matched with input data (describing the motion tasks and people) to estimate the value (weight) of data links in a prescribed network. Once the network weighting values are established for one set of data, the network can be used, when given new input data, to predict appropriate body movements.

4. Weighted pseudoinverse method for reach modeling in which the trajectory of the hand is related to changes in joint angles by minimizing a prescribed cost function, which in Zhang and Chaffin (2000) was the weighted kinetic energy imparted by the movement of each body segment required to guide the hand along the prescribed path.

All of these methods can produce realistic motions, but most have been tested with limited motion databases, and for a small number of linkage systems, normally three to 
five links. Some of these models become quite computationally complex and are thus not appropriate at this time for real-time simulations. Also, several human motion researchers have reported that people develop a very repeatable behavior in reaching motions, but this pattern is quite specific to a particular person (Latash, 1998). Such individual variation is difficult to accommodate in many of the existing models.

After reviewing the various models and their validation, it was decided by our research group to use an old approach, gather lots of motion data from a diverse population and use a sophisticated statistical model to determine the most prominent sources of variation in the data, and then develop prediction models that would account for these effects. To do this, two very important technical developments were necessary. One, a laboratory was needed that could measure accurately the three-dimensional motions of a variety of participants when performing common tasks; and two, a statistical model had to be developed that was capable of handling very large and dense three-dimensional data sets. What follows is a brief description of both of these technologies.

\section{METHODS FOR STUDYING AND MODELING HUMAN REACHES}

\subsection{Reach Motion Capture Laboratory}

The following is a description of the history and current use of our Human Motion Simulation Laboratory.

1994 Vehicle Seated Reach Study. In 1994, a four-camera motion capture laboratory was created within the Center for Ergonomics to study simulated vehicle driving reaches. A stationary driving simulator was used. Thirty-eight people of both genders and ages 18 to 72 were recruited. While driving the simulator down a slowly turning road they were asked periodically to reach to 52 targets placed around them. Reflective markers were tracked, and a four-link torso, shoulder, arm, and forearm-hand linkage was used to derive seven body angles during the motions. About 4,000 motions were recorded. Modeling of these data are reported in Zhang and Chaffin (2000), and in Chaffin, Faraway, Zhang, and Woolley (2000).

1998 Seated Reach Study. During the summer of 1998, we recruited 20 men and women (age 18 to 74 ) to perform seated reaching motions. The motions began with either their hands resting in front of them or from a 10:00-2:00 position on a steering wheel. The reaches were performed with the right hand moving to between 40 and 52 targets widely dispersed around them. Three different seat types were used: a car seat, a light truck seat, and an industrial seat. Some of the motions required moving a light hand load. About 8,000 motions were recorded. A seven-link system was created to model the data. Nineteen angles of the linkage were used for subsequent statistical modeling. A graphic package was derived to visually scan the motions and allow us to verify the completeness of each motion data set. This same graphic package was then used to visually validate the outputs from the statistical motion predictions.

The results of these two studies indicated that seated people are relatively consistent when repeating a variety of reaches, and that our statistical model to describe the motions captures about $97 \%$ of the between participant variation. We also found that a person's stature is the major personal attribute affecting motions, followed by one's age, and then gender. The presence of a light load did not have a great effect on the motions, but the 
type of seat did affect the degree to which the torso moves to accommodate certain upper limb and shoulder motions.

1999 Motion Capture Study. During the summer of 1999, we undertook a much larger motion capture study. Once again we recruited 20 men and women (ages 21 to 68 years). The conditions for this study included both seated and standing motions, reaching with one and both hands, moving heavier objects than the previous study, and reaching over and under barriers.

A full-body 17-link kinematics system was used to develop estimates of 45 local and 45 global joint angles. A total of 17,000 motions was recorded using our upgraded motion capture laboratory, which allows us to acquire data from both six cameras and six magnetic motion sensors simultaneously. We also recorded psychophysical exertion ratings for each motion.

Because of the complexity of these data, a great deal of effort has been devoted this past year to verifying and organizing the data for statistical analysis.

2000 Motion Capture Study. One of the major determinates of a person's whole body motion is the orientation of the hand at the end of a motion. The orientation of the hand is often forced into some extremely awkward postures when reaching into an enclosed space (e.g., glove box), or when reaching to activate a control surface (e.g., parking brake handle), or when picking up or using a hand tool (e.g., using a drill to fasten pieces lying on a bench). The resulting motions of the entire body are driven to some extent by the location of the hand, the orientation of the hand, and any forces that may be required at the termination of a motion.

Because of these complexities a series of new studies was undertaken. We recruited 22 men and women (ages 19 to 79). The participants were seated and asked to reach to 60 different target locations and grasp a small block. The orientation of the grasp was varied with each reach, so that the hand was forced into sometimes extreme wrist and hand postures. While grasping the block they would then have to push or pull it with about 3 pounds of force.

These activities produced about 9,000 motions. Exertion ratings were also obtained. Verification of these data has been completed and motion modeling is beginning.

\subsection{Overview of Motion Modeling}

Why model motion data? There are three major reasons why modeling of motion data is necessary, instead of using the motion data directly to drive a digital human animation.

- Modeling can reveal patterns in complex data that are not readily apparent, and thus the factors that most affect the general form of the data can be inferred. This can lead to a more complete understanding of the underlying motor behavioral strategies and biomechanics that people use to control motions.

- Modeling allows the prediction of how different groups of people move; that is, it provides a necessary distinction in motions between subgroups for different tasks.

- Modeling provides the ability to predict motions under conditions that are different than studied (i.e., one can extrapolate the data to analyze novel situations). 


\subsection{Modeling of Motions in HUMOSIM Laboratory}

4.3.1. Function Regression Method for Reach Modeling. If one does not wish to specify the intended hand motion trajectory, but rather has only a starting posture and an ending target location to which the hand is to reach, then a general whole body motion prediction approach is required. As discussed earlier, inverse kinematics with optimization criteria can be used but are computationally difficult (time consuming) and may not result in appropriate motions because the optimization criteria that guide coordinate movements would appear to be very dependent on specific situations.

It is for these reasons that we have chosen to develop a very efficient empirical method. It is referred to as the functional regression method for predicting joint angles during the motion of a hand to a specific target. It is particularly useful in dynamic motion modeling wherein three-dimensional D motion capture technologies can rapidly produce very large and dense data sets that are not very noisy throughout a motion (i.e., the joint angles $\theta(t)$ are smooth, regular and known).

Faraway (1997) has developed a functional regression model (1) for this purpose. It uses the form:

$$
\begin{aligned}
\theta(t)= & \beta_{0}(t)+C_{\chi} \beta_{\chi}(t)+C_{y} \beta_{y}(t)+C_{z} \beta_{z}(t) \\
& +C_{\chi} C_{y} \beta_{\chi y}(t)+C_{y} C_{z} \beta_{y z}(t)+C_{z} C_{\chi} \beta_{z \chi}(t) \\
& +C_{\chi}^{2} \beta_{\chi}^{2}(t)+C_{y}^{2} \beta_{y}^{2}(t)+C_{z}^{2} \beta_{z}^{2}(t)+D
\end{aligned}
$$

where

$$
\begin{aligned}
\theta(t)= & \text { predicted joint angles over time, } \\
C_{\chi}, C_{y}, \text { and } C_{z}= & \text { target coordinates, } \\
\beta(\mathrm{t})= & \text { parametric functions to be estimated, and } \\
D= & \text { demographic variables (e.g., age, stature, gender, etc.) that could } \\
& \text { modify the predictions. }
\end{aligned}
$$

Note that the model shown is a quadratic model, which was found by Faraway (1997) to account for approximately $80 \%$ of the joint angle deviations measured in one early set of reach data.

One of the nice aspects of this type of modeling is that eigen functions can be derived easily, which provides a means to measure how well a particular model fits a set of data at various points during a motion. It also provides a means to place confidence limits around the $\theta(t)$ predictions so that data from different experimental conditions and populations can be statistically compared. An example of this is shown in Figure 3, which shows the elbow included angles for a group of participants reaching from the steering wheel to an area at the far right side of a radio located in the center of the instrument panel.

One of the limitations in this particular empirical approach is that it models the motion postures only in terms of angles, so that when the terminal posture is reconstructed using conventional forward kinematics, the hand may not coincide with the reach target. This is due to variation in individual link lengths, the necessarily approximate nature of the model, and variation in reaching behavior. This problem has been attacked by adding an inverse 


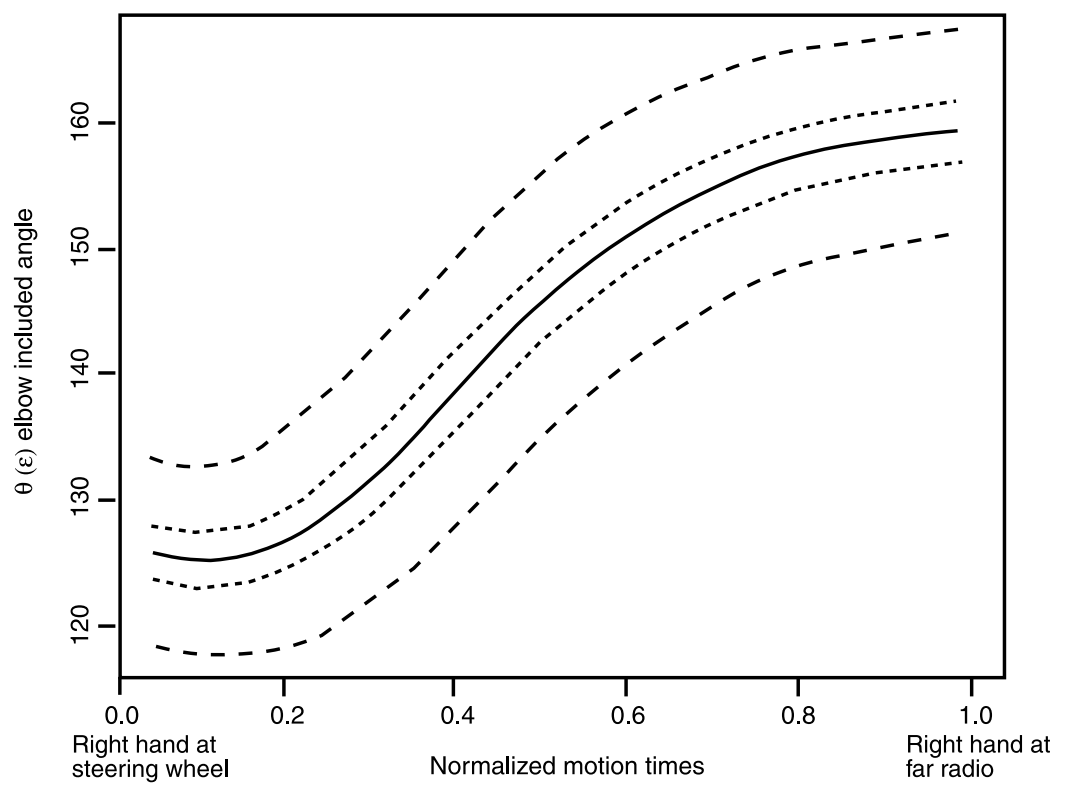

Figure 3 The predicted elbow angle response (solid line) for a reach to a new target situated between the radio and the glove box, with $95 \%$ simultaneous confidence bands for the mean response (inner pair) and for a new observation (outer pair) also shown (Faraway, 1997).

kinematic rectification scheme to the empirical position predictions (Faraway, Zhang, \& Chaffin, 1999). The present version resolves the usual redundancy in inverse kinematic predictions by choosing the posture that comes as close as possible to angle-only model predictions while satisfying the hand on the target constraint. Closeness is defined by a metric that takes account of the varying accuracies among the angles that define the posture. This rectification scheme ensures that the hand is on the target in the final posture, but it necessarily involves changes in the originally predicted locations of the elbow and shoulder. These changes are usually improvements, as demonstrated in predictions described in Faraway, Zhang, and Chaffin (1999). The amount of improvement to be expected depends on many things, but in a couple of typical cases, the error at the shoulder was reduced by an average $20 \%$ and the elbow by an average $50 \%$.

A further improvement in the angle predictions is being considered by our group. This method performs the rectification by minimizing the Euclidean distances between the angular velocity trajectories of the empirically predicted and the rectified motion. Preliminary results indicate that such a procedure produces smooth motions (no discontinuities), is computationally efficient, and ensures that the hand ends at the intended target (Faraway \& Hu, 2001).

4.3.2. Motion Engineering Algorithm Development. Though our functional regression method has performed very well in modeling many different types of motions, we believe it is necessary to have the ability to predict motions associated with unexpected motion conditions. An example of this would be when a designer needs to synthesize motions of various people that would allow them to move around obstacles. In 
such a scenario, one could envision myriad different obstacles that exist and impede normal motions in everyday manual tasks. Though we have studied some motions that were performed by participants with obstacles present, the form and number of such experiments, relative to the number that would be needed for all different types of barrier-type motions, have been very limited. Therefore, one of our group, Woojin Park, has been working on methods and algorithms to extract from our existing database those motions that approximate motions probably used by a person when avoiding an obstacle, and then modifying and assembling these into a synthetic obstacle avoidance motion that still retains the basic kinematics of the original motion. To accomplish this, three development steps are involved:

- Motion coding - a method for identifying motions in the database that are similar to those desired so that they can be easily identified in the database and used as a "root" motion to be modified to represent the more complicated obstacle avoidance motions.

- Motion search-an efficient method for searching in the database for motions that are structurally the same to serve as the "root" motions needed to create a specific obstructed motion.

- Motion modification and assimilation - the methods used to slightly change or morph a root motion and combine it with other root motions to create a new synthesized obstacle avoidance motion. This latter method is described by Park, Chaffin, and Martin (2001).

\section{HUMOSIM BIOMECHANICS AND EXERTION RESEARCH}

The procedures described above provide a means to efficiently estimate values for the joint kinematics involved in normal reaching behaviors. These kinematic values, when combined with a good DHM as shown in Figure 1, provide an improved means of assessing dynamic population fit/clearance, reach, and visibility requirements.

In addition, within the Human Motion Simulation HUMOSIM Laboratory, we have linked an existing biomechanical model to the new motion kinematic model. The model presently linked is the University of Michigan 3D Static Strength Prediction Program ${ }^{\mathrm{TM}}$. This provides a prediction of population muscle static strength requirements at each joint throughout the movement. In addition, estimates of lumbar motion segment static compression and shear forces are developed. The joint strength requirements and spinal compression forces can then be compared to NIOSH limits for injury risk analysis. The general logic for these combined programs is illustrated in Figure 4.

The combined HUMOSIM motion models and biomechanical models will not only provide a better scientific basis for why certain types of extreme reach and object motion conditions should be avoided, such as those now flagged as being hazardous by some ergonomics posture checklists, but they will allow the development of a new set of dynamic functional reach envelopes. In other words, we believe that both the existing posture checklists and available reach envelopes greatly oversimplify the true basis for determining what types of upper limb and whole body motions people can perform safely. We acknowledge that such simplifications were necessary and useful in the past for performing paper-and-pencil assessments of a work space. But with the development of DHM methods in CAE applications today, the underlying simplifying assumptions must be questioned. We are proposing to do this with three different but complementary activities. 


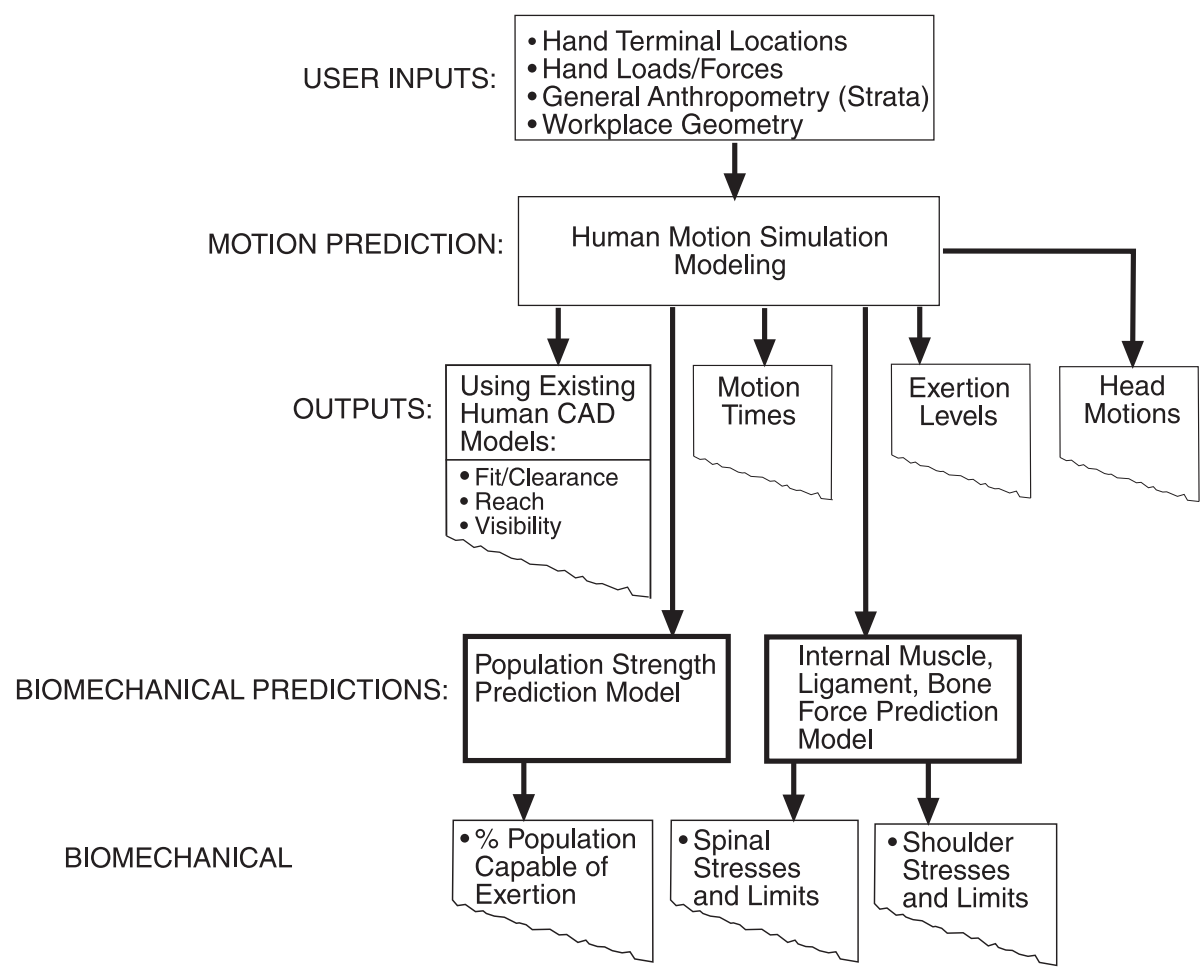

Figure 4 Diagram of University of Michigan's HUMOSIM software project.

First, there is the continuing analysis and statistical modeling of the extensive psychophysical exertion data we have acquired. In both the 1999 and 2000 reach studies, we have asked the participants after each reach task to rate on a scale of 1 to 10 the effort level of the prior motion. Low back, shoulder, and now wrist/lower arm exertion ratings have been obtained. A preliminary analysis of some of these data is reported in Kim, Martin, Chaffin, and Woolley (2001).

Second, there is no doubt that the complex and highly mobile shoulder joint dictates much of the reach capability of a seated person. Also, the shoulder is a joint(s) that can be easily injured during certain types of dynamic arm motions. There do exist several static biomechanical models of the shoulder complex, but it is not clear that these are appropriate or robust enough for the types of dynamic conditions we have documented. We therefore are developing a new dynamic shoulder model to predict critical biomechanical stresses within select shoulder tissues. This has required (a) the development of a threedimensional dynamic upper limb model of the moments and forces at the shoulder, which works directly from our motion database, (b) review of existing static shoulder biomechanical models, (c) development of a new shoulder model that represents muscle and other tissue geometric and physiological changes during movements, (d) performance of a sensitivity analysis to determine which model outputs are most sensitive to motion input values, (e) comparison of the model outputs with exertion ratings and EMG values to validate the muscle force predictions of the model, and (f) development of a graphical interface to communicate model outputs for assistance in determining acceptable and 
unacceptable reach requirements in a task. Some of the initial work on this topic is described in Dickerson and Chaffin (2001).

Though most models of human motion have concentrated on understanding the kinematics involved in hand motions, the need to understand the kinematics of head positioning and movement is also being studied. This problem has become quite well recognized in the automobile industry lately as people move their head and eyes away from the roadway while driving to dial a phone number or to interact with their navigation or entertainment systems. Current work is modeling the dynamics of head motions as a person reaches to various targets. Does the head move in certain ways to assist in guiding the hand throughout the motion, or does it simply assist in the final hand motions? And what visual requirements may alter head motions during reaching tasks? Some of this work is described in Kim and Martin (2001).

Lastly, we are very concerned that special populations of individuals may have dynamic reach capabilities that are quite different than now published in some ergonomics guides for the handicapped. At present it is estimated that about 3 million people in the United States between 18 and 64 years of age use an ambulatory aid (e.g., cane walker or wheelchair), and this does not include people with chronic low back pain. With financial assistance from the National Institute for Disability Research and Rehabilitation, we are undertaking a 3-year study of people who have suffered spinal cord injuries and are paraplegic, and people who suffer chronic low back pain. In both groups, when performing seated reaches, their reaching kinematics and capabilities are expected to be different than those without such impairments. In addition, we expect the coordination of muscle actions to be altered. By measuring the EMG signals from select torso muscles in both affected groups and in a healthy group, we expect to be better able to understand the basis for the altered motions and limitations from these conditions. An initial report of some of these changes is in Womack, Kim, Martin, Haig, and Chaffin (2001).

In summary, these three activities, (a) modeling of psychophysical effort ratings, (b) modeling of shoulder muscle biomechanics, and (c) evaluating motions and EMGs of persons with spinal cord injuries and low back pain, will provide a comprehensive understanding of reach behaviors and limitations in a variety of populations.

\section{SUMMARY OF PROGRESS ON STUDYING AND MODELING REACH MOTIONS}

The field of ergonomics has progressed very well with the aid of static models of people performing various manual tasks. Yet it is clear that there is a limit to what these static models can do to help us fully understand the mechanisms of injury to the musculoskeletal system, as well as aid us in visualizing the way people might actually behave within a virtual workplace or virtual vehicle.

The HUMOSIM Laboratory has been established to provide the fundamental knowledge and data needed to simulate realistic human motions in a large variety of common tasks. The models we are producing will enhance existing commercial, digital human software packages for CAE and CAD projects. We believe that once human motions are rendered in these systems, human-centered ergonomic design methods will be facilitated and used much more than is the case today. Though much has been done in the last few years in this regard, much remains to be done. Hopefully this article will serve to describe what can be done, but will also serve to stimulate others to join in this exciting field. 


\section{ACKNOWLEDGMENTS}

The authors wish to thank the following organizations for sponsoring this work: General Motors, Ford, DaimlerChrysler, US Army (TACOM), Johnson Controls Inc. International Truck, TRW Foundation, the UM Automotive Research Center, and the National Institute for Disability Rehabilitation and Research.

\section{REFERENCES}

Allard, P., Cappozzo, A., Lundberg, A., \& Vaughan, C.L. (Eds.). (1998). Three-dimensional analysis of human locomotion. West Sussex, England: J. Wiley \& Sons Ltd.

Chaffin, D.B. (Ed.). (2001). Digital human modeling for vehicle and workplace design. Warrendale, PA: Society of Automotive Engineers.

Chaffin, D.B., Faraway, J.J., Zhang, X., \& Woolley, C. (2000). Stature, age, and gender effects on reach motion postures. Human Factors, 42(3), 408-420.

Dickerson, C.R., \& Chaffin, D.B. (2001). Exertion-driven strength modeling of the shoulder. Presented at SAE Digital Human Modeling Conference, Arlington, VA, June 26-28.

Faraway, J.J. (1997). Regression analysis for a functional response. Technometrics, 39(3), $254-262$.

Faraway, J.J., \& Hu, J. (2001). Modeling variability in reaching motions. Accepted for Presentation at SAE Digital Human Modeling Conference, Arlington, VA, June 26-28.

Faraway, J.J., Zhang, X., \& Chaffin, D. (1999). Rectifying postures reconstructed from joint angles to meet constraints. Journal of Biomechanics, 32, 733-736.

Feyen, R., Liu, Y., Chaffin, D., \& Jimmerson, G. (2000). Computer-aided ergonomics: A case study of incorporating ergonomics analyses into workplace design. Applied Ergonomics, 31, 291-300.

Feyen, R., Liu, Y., Chaffin, D., Jimmerson, G., \& Joseph, B. (1999). New software tools improve workplace design. Ergonomics in Design, April, 24-30.

Kim, K., \& Martin, B. (2001). Prediction of head orientation based on the visual image of a three dimensional space. Presented at SAE Digital Human Modeling Conference, Arlington, VA, June 26-28.

Kim, K., Martin, B.J., Chaffin, D.B., \& Woolley, C.B. (2001). Modeling of effort perception in lifting and reaching tasks. Presented at SAE Digital Human Modeling Conference, Arlington, VA, June 26-28.

Latash, M.L. (Ed.). (1998). Progress in motor control. Champaign, IL: Human Kinetics.

Nigg, B.M., MacIntosh, B.R., \& Mester, J. (Eds.). (2000). Biomechanics and biology of movement. Champaign, IL: Human Kinetics.

Park, W., Chaffin, D.B., \& Martin, B.J. (2001). Modifying motions for avoiding obstacles. Presented at SAE Digital Human Modeling Conference, Arlington, VA, June 26-28.

Womack, N., Kim, K., Martin, B., Haig, A., \& Chaffin, D. (2001). Analysis and simulation of upper body motion of patients affected by low back pain or spinal cord injury Presented at the RESNA 2001 Annual Conference and Educational Program, Reno, NV, June 22-26.

Zatsiorsky, V.M. (1998). Kinematics of Human Motion. Champaign, IL: Human Kinetics.

Zhang, X., \& Chaffin, D. (2000). A three-dimensional dynamic posture prediction model for simulating in-vehicle seated reaching movements: Development and validation. Ergonomics, 43(9), 314-1330. 